\title{
Solution-Phase Singlet Fission in a Structurally Well-Defined Norbornyl-Bridged Tetracene Dimer - Supporting Information
}

\section{Jasper D. Cook, Thomas J. Carey, and Niels H. Damrauer*}

Department of Chemistry and Biochemistry, University of Colorado, Boulder Colorado 80309, United States

*author to whom correspondence should be addressed

\section{Table of Contents}

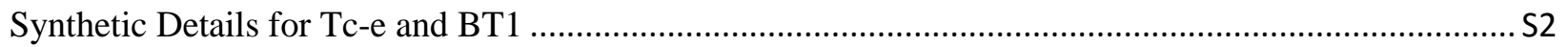

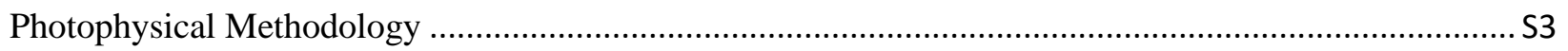

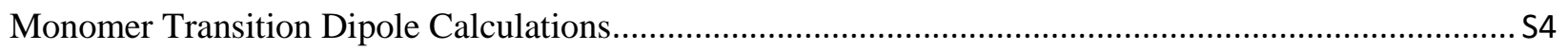

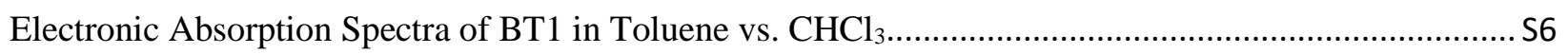

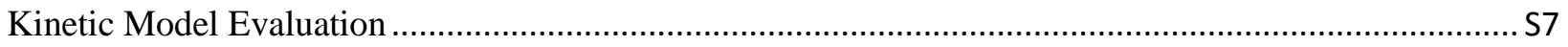

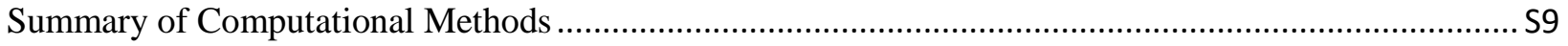

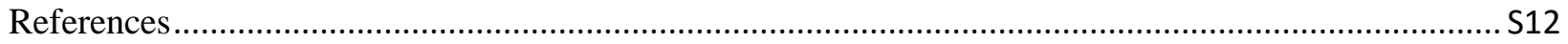




\section{Synthetic Details for Tc-e and BT1}

The general synthetic approach and data are covered in a separate work. ${ }^{1}$ A brief summary is provided here. Dimer (BT1) material studied in this work was synthesized as depicted in Scheme S1, while Monomer (Tc-e) material was synthesized according to the procedure shown in Scheme S2.

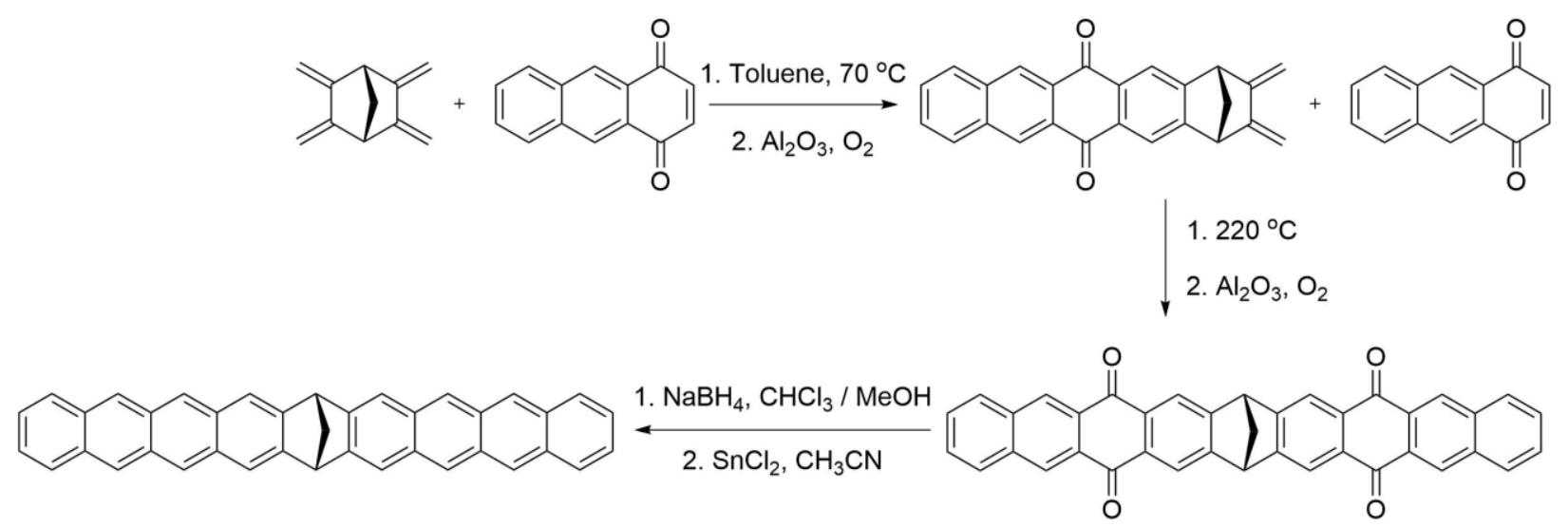

Scheme S1. Synthetic method used to generate the BT1 used in this work.
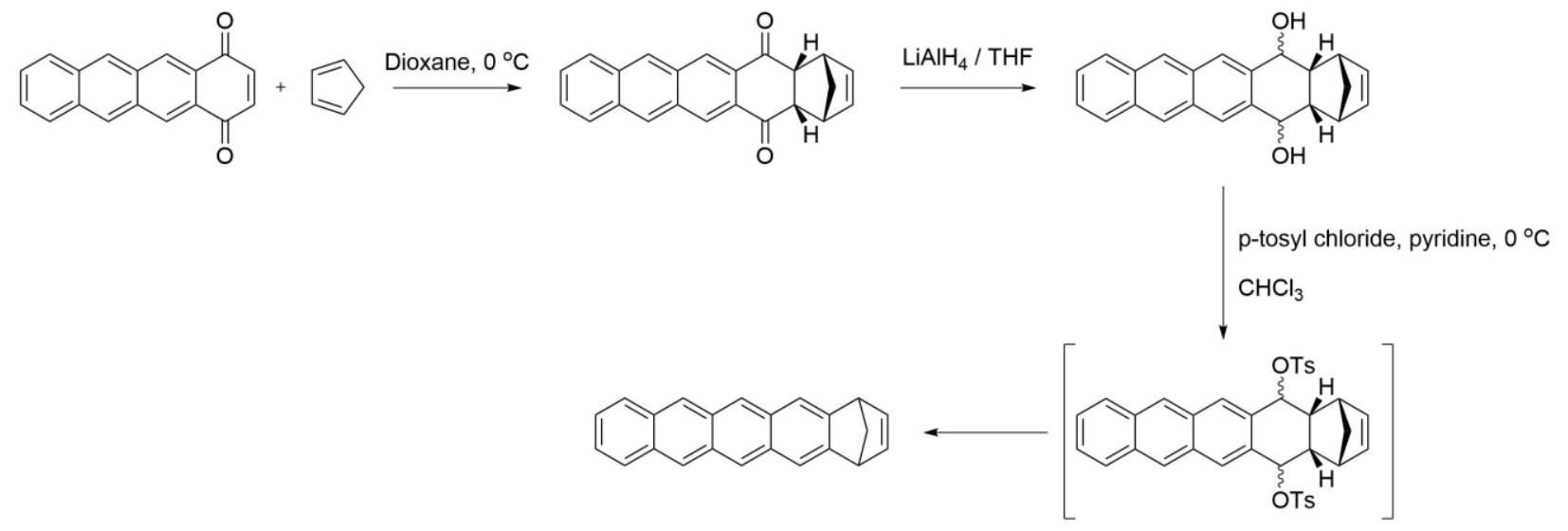

Scheme S2. Synthetic method used to create monomer Tc-e used in this work. 


\section{Photophysical Methodology}

All photophysical measurements were carried out in $1 \mathrm{~cm}$ quartz cuvettes on samples that had been de-aerated by bubbling with argon for 20 minutes. Absorption spectra were acquired with a Cary 5000 UV-Vis-NIR spectrophotometer. Steady-state emission spectra were acquired using an Olis SLM fluorometer, and fluorescence quantum yields were measured relative to Coumarin 480 in methanol $(\Phi e m=0.87)$. Time-resolved emission decays were obtained using an $82 \mathrm{MHz}$ mode-locked Ti:sapphire oscillator (Tsunami, Spectra-Physics) stepped down to a repetition rate of $4.1 \mathrm{MHz}$ using a NEOS Technologies pulse-picker. The second harmonic (395nm) was generated with a $\beta$-barium borate crystal and filtered to remove residual fundamental before exciting the sample (fluence $\sim 2 \mu \mathrm{J} / \mathrm{cm}^{2}$ ). Emission was collected at magic angle $\left(54.7^{\circ}\right.$ ), passed through a monochromator (Oriel 77250), detected with a Hamamatsu R3809U-50 microchannel plate detector, and analyzed with a Becker-Hickl time-correlated single photon counting card. The instrument response was convoluted with a sum of exponentials to create the fits in Figure 4a (main text). 


\section{Monomer Transition Dipole Calculations}

To describe the UV-vis transitions observed, transition dipole moments were calculated for the first three observed singlet electronic transitions $\left(S_{1} \leftarrow S_{0}, S_{2} \leftarrow S_{0}\right.$, and $\left.S_{3} \leftarrow S_{0}\right)$ using only the frontier orbital space of Tc-e consisting of its HOMO-1, HOMO, LUMO, and LUMO+1 orbitals. The $\mathrm{S}_{1} \leftarrow \mathrm{S}_{0}$ (the ${ }^{1} \mathrm{~L}_{\mathrm{a}}$ band in Platt's notation ${ }^{2}$ ) can be well described as arising from a HOMO $\rightarrow$ LUMO orbital transition. The $\mathrm{S}_{2} \leftarrow \mathrm{S}_{0}$ and $\mathrm{S}_{3} \leftarrow \mathrm{S}_{0}$ excitations are, respectively, accessed by inphase and out-of-phase combinations of the HOMO-1 $\rightarrow$ LUMO and HOMO $\rightarrow$ LUMO+1 transitions. The lower-energy, out-of-phase combination is only weakly allowed while the higher-energy, in-phase combination is strongly allowed. The frontier orbitals considered are depicted in Figure S1.

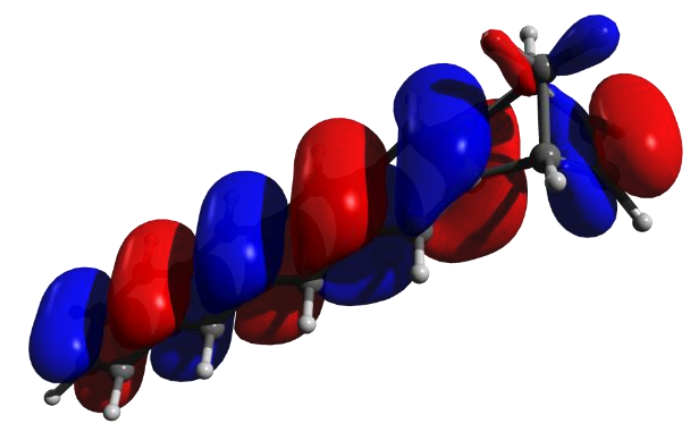

HOMO-1

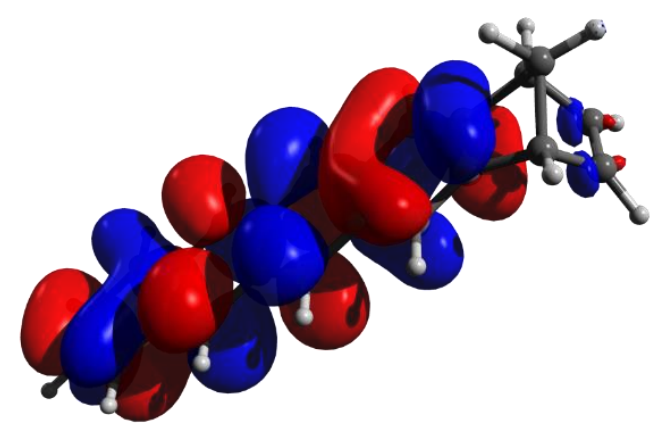

LUMO

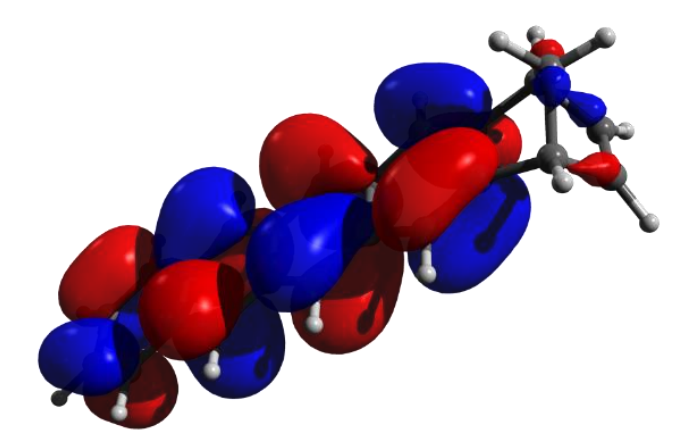

HOMO

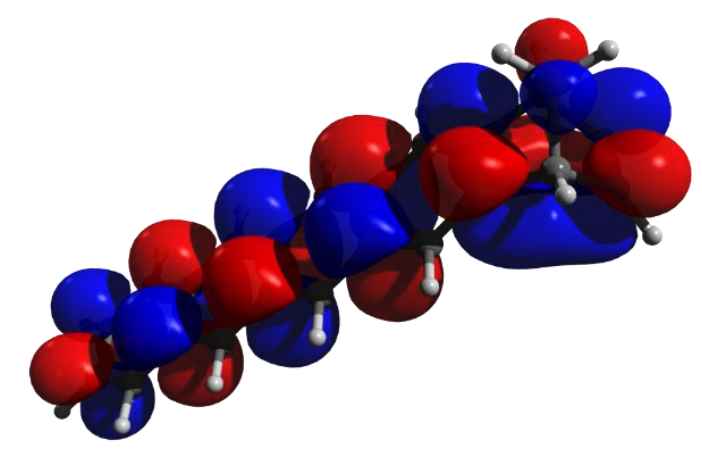

LUMO+1

Figure S1. Frontier orbitals of Tc-e. These were obtained previously ${ }^{3}$ following geometry optimization of Tc-e with DFT using the range-corrected $\omega$-B97XD density functional and the $6-31 G(d)$ basis set. 
Transition dipole moments $\overrightarrow{D_{l j}}$ between orbitals $\Psi_{i}$ and $\Psi_{j}$ were computed numerically according to the following sums (Equations S1-4):

$$
\begin{aligned}
& D_{i j, x}=\sum_{x} \Psi_{i} x \Psi_{j} \\
& D_{i j, y}=\sum_{y} \Psi_{i} y \Psi_{j} \\
& D_{i j, z}=\sum_{z} \Psi_{i} z \Psi_{j} \\
& \overrightarrow{D_{l \jmath}}=D_{i j, x} \hat{x}+D_{i j, y} \hat{y}+D_{i j, z} \hat{z}
\end{aligned}
$$

Here, orbital wavefunctions $\left(\Psi_{i}\right.$ and $\Psi_{j}$ ) are the frontier orbitals from HOMO-1 to LUMO+1 (see Fig. S1) obtained (previously) from geometry optimization without symmetry constraints of Tc-e with DFT using the range-corrected $\omega$-B97XD density functional and the 6-31G(d) basis set using the Gaussian 09 software package. ${ }^{3}$ Output cube files were parsed and multiplied on an element-by-element basis with the corresponding position vector element, then scaled by the

\begin{tabular}{|c|c|c|c|c|}
\hline $\begin{array}{l}\text { Electronic } \\
\text { Transition }\end{array}$ & $\begin{array}{l}\text { Transition } \\
\text { Name (Platt) }\end{array}$ & Orbital Origins & $\begin{array}{l}\text { Transition } \\
\text { Dipole /e } \AA\end{array}$ & $\begin{array}{l}\text { Polarization } \\
\text { Axis }\end{array}$ \\
\hline $\mathrm{S}_{1} \leftarrow \mathrm{S}_{0}$ & ${ }^{1} \mathrm{~L}_{\mathrm{a}}$ & $\mathrm{HOMO} \rightarrow$ LUMO & 1.6 & Short \\
\hline $\mathrm{S}_{2} \leftarrow \mathrm{S}_{0}$ & ${ }^{1} \mathrm{~L}_{\mathrm{b}}$ & $\begin{array}{l}(\mathrm{HOMO}-1 \rightarrow \mathrm{LUMO}) \\
- \\
(\mathrm{HOMO} \rightarrow \mathrm{LUMO}+1)\end{array}$ & 0.08 & Long \\
\hline $\mathrm{S}_{3} \leftarrow \mathrm{S}_{0}$ & B & $\begin{array}{l}(\mathrm{HOMO}-1 \rightarrow \mathrm{LUMO}) \\
+ \\
(\mathrm{HOMO} \rightarrow \mathrm{LUMO}+1)\end{array}$ & 5.98 & Long \\
\hline
\end{tabular}
appropriate voxel volume and summed. The results for the transition dipoles $\overrightarrow{D_{\imath \jmath}}$ are summarized in Table S1.

Table S1. Transition dipole moments for the first 3 singlet transitions in Tc-e 


\section{Electronic Absorption Spectra of BT1 in Toluene vs. $\mathrm{CHCl}_{3}$}

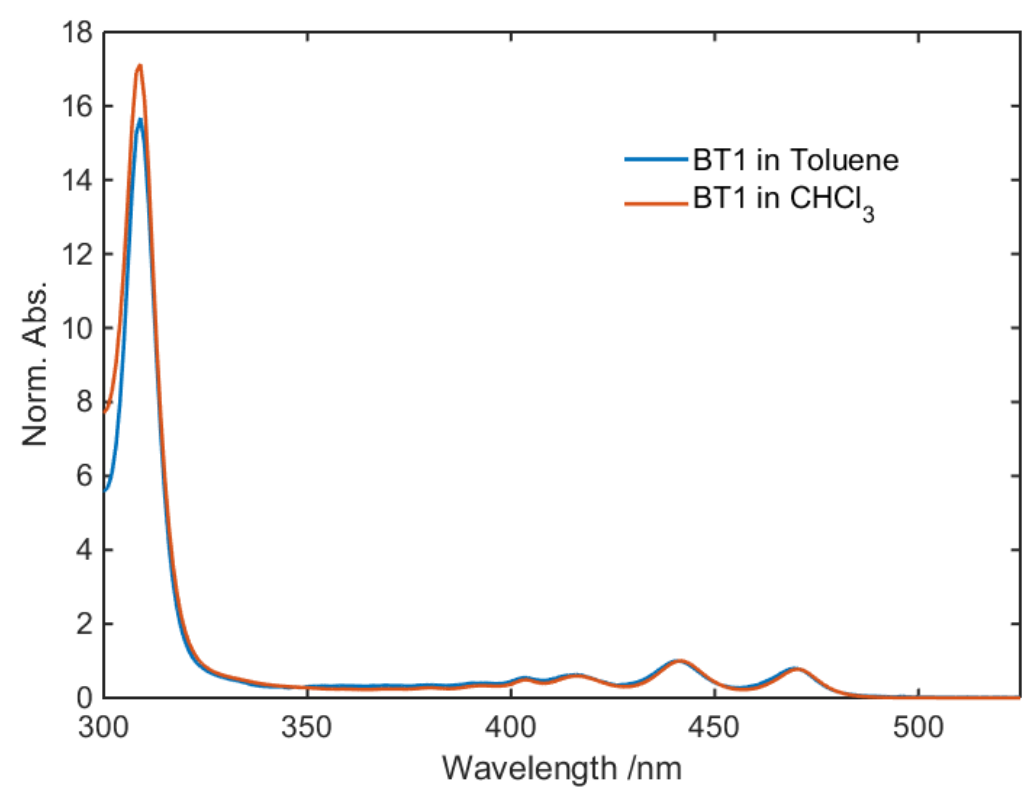

Figure S2. Solvent-dependence of BT1 electronic absorption in toluene vs. $\mathrm{CHCl}_{3}$ normalized according to the $\mathrm{S}_{1}$ absorbance. 


\section{Kinetic Model Evaluation}

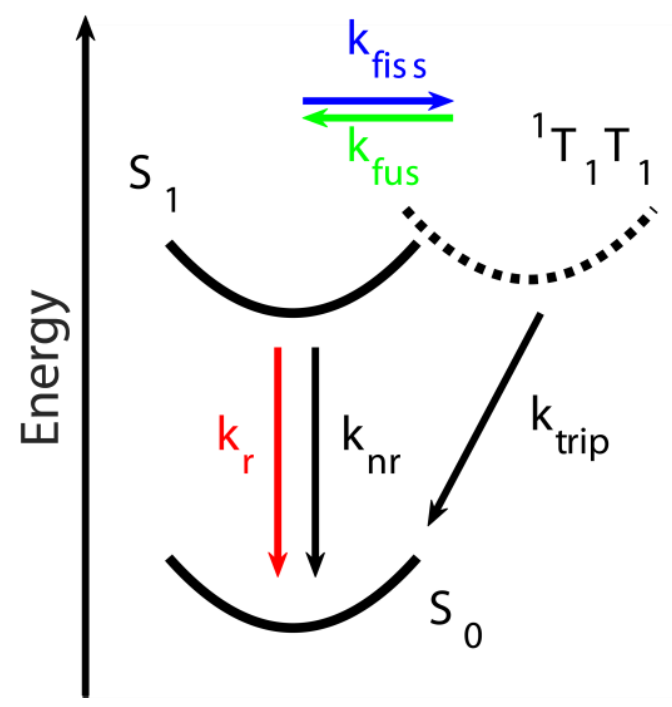

Figure 4b (reproduced here). Cartoon depicting the kinetic model used to describe the behavior of BT1 following excitation.

The kinetic model in Fig. 4b (main text; reproduced above) yields the differential equations 1 and 2 in the manuscript and shown below, where $k_{l o s s}=k_{r}+k_{n r}$. Assumptions relevant for solving these are detailed in the main text.

$$
\begin{aligned}
& \frac{d S_{1}}{d t}=-\left(k_{\text {loss }}+k_{f i s s}\right) S_{1}+k_{f u s}{ }^{1} T T \\
& \frac{d^{1} T T}{d t}=k_{f i s s} S_{1}-\left(\frac{k_{\text {trip }}}{2}+k_{f u s}\right)^{1} T T
\end{aligned}
$$

In matrix form, this system of differential equations can be rewritten as:

$$
\overrightarrow{\boldsymbol{N}}^{\prime}(t)=\overline{\bar{K}} \overrightarrow{\boldsymbol{N}}(t)
$$

Where the vector $\mathbf{N}$ consists of the populations of the states in question, here taken to be $S_{1}$ and ${ }^{1} \mathrm{TT}$, and the matrix $\mathbf{K}$ holds the relevant decay rates, and is equal to: 


$$
\overline{\overline{\boldsymbol{K}}}=\left(\begin{array}{cc}
-\left(k_{\text {loss }}+k_{f i s s}\right) & k_{f u s} \\
k_{f i s s} & -\left(\frac{k_{\text {trip }}}{2}+k_{f u s}\right)
\end{array}\right)
$$

General solutions to equation S6 are well-known to be $n$-exponential (where $n$ is the size of square matrix $\boldsymbol{K}$; here $n=2$ ) with the following form:

$$
\overrightarrow{\boldsymbol{N}}(t)=c_{1} e^{\lambda_{1} t} \overrightarrow{\boldsymbol{V}_{\mathbf{1}}}+c_{2} e^{\lambda_{2} t} \overrightarrow{\boldsymbol{V}_{\mathbf{2}}}
$$

Scalars $\lambda_{i}$ and vectors $\boldsymbol{V}_{i}$ are, respectively, the $i^{\text {th }}$ eigenvalues and $i^{\text {th }}$ eigenvectors of matrix $\boldsymbol{K}$. The TCSPC observable (the population of $S_{1}$, or $\mathbf{N}_{1}$ from equation (5)) is given by:

$$
S_{1}(t)=c_{1} V_{11} e^{\lambda_{1} t}+c_{2} V_{12} e^{\lambda_{2} t}=A_{1} e^{-t / \tau_{1}}+A_{2} e^{-t / \tau_{2}}
$$

The observed lifetimes $\tau_{i}$ and amplitudes $A_{i}$ are therefore equal to:

$$
A_{1}=c_{1} V_{11}, A_{2}=c_{2} V_{12}, \tau_{1}=-\frac{1}{\lambda_{1}}, \tau_{2}=-\frac{1}{\lambda_{2}}
$$

Diagonalizing $\mathbf{K}$ gives the desired eigenvalues $\lambda_{i}$ and eigenvector elements $V_{j i}$, and application of initial conditions gives the coefficients $c_{i}$.

$$
\begin{gathered}
\tau_{1,2}=-\frac{1}{\lambda_{1,2}}= \\
\frac{-4}{-2 k_{f i s s}-2 k_{f u s}-2 k_{\text {loss }}-k_{\text {trip }} \mp \sqrt{\left(2 k_{f i s s}+2 k_{f u s}+2 k_{\text {loss }}+k_{\text {trip }}\right)^{2}-8\left(2 k_{f u s} k_{\text {loss }}+k_{\text {fiss }} k_{\text {trip }}+k_{\text {loss }} k_{\text {trip }}\right)}} \\
\overrightarrow{\boldsymbol{N}}(0)=\left(\begin{array}{l}
1 \\
0
\end{array}\right)=c_{1} \overrightarrow{\boldsymbol{V}_{1}}+c_{2} \overrightarrow{\boldsymbol{V}_{2}} \Longrightarrow \\
c_{1,2}=\mp \frac{2 k_{\text {fiss }}}{\sqrt{\left(2 k_{\text {fiss }}+2 k_{f u s}+2 k_{\text {loss }}+k_{\text {trip }}\right)^{2}-8\left(2 k_{f u s} k_{\text {loss }}+k_{f i s s} k_{\text {trip }}+k_{\text {loss }} k_{\text {trip }}\right)}}
\end{gathered}
$$

Given an observed $A_{1}$ and $A_{2}$, with corresponding $\tau_{1}$ and $\tau_{2}$, this system of equations was solved numerically to obtain values for $k_{f i s s}, k_{f u s}$, and $k_{\text {loss. }}$. The radiative and nonradiative rate constants $k_{r}$ and $k_{n r}$ were separated from $k_{\text {loss }}$ using the quantum yield.

$$
\Phi_{e m}=\frac{k_{r}}{k_{r}+k_{n r}}=\frac{k_{r}}{k_{\text {loss }}}
$$

As noted in the main text, we have taken advantage of the fact that $k_{\text {trip }}$ is insignificant and that any losses of $\mathrm{S}_{1}$ population due to $k_{\text {fiss }}$ are regained by $k_{\text {fus }}$. In the case of the monomer, $k_{\text {loss }}$ is simply equal to $k_{o b s}$, or $1 / \tau_{\text {obs. }}$. 


\section{Summary of Computational Methods}

Single point calculations $(6-3 \lg (\mathrm{d})$ basis, the $\omega-\mathrm{B} 97 \mathrm{XD}$ range corrected density functional, and gas phase) were carried out using the Gaussian 09 software package (Revision D.01) running on the National Energy Research Super Computer (NERSC) facility (www.NERSC.gov). ${ }^{4}$ Two relevant geometries were explored whose Cartesian coordinates were previously determined ${ }^{3}$ using $\triangle \mathrm{SCF}$ and TD-DFT with gradients $(6-31 \mathrm{~g}(\mathrm{~d})$ basis, the $\omega$-B97XD range corrected density functional, and a polarizable continuum model parameterized for toluene). The first geometry (A) is that of the localized singlet excited state referred to as $\mathrm{S}_{1-\mathrm{Loc}}$ in our previous work. ${ }^{3}$ The second geometry (B) is that of the optimized quintet state Q. ${ }^{3}$ A schematic showing these geometries on a reaction coordinate diagram is provided below (Scheme S3). Using gas-phase single point energy calculations, the difference in energy of the quintet state Q calculated at geometry single points $A$ and B provides a measure of the inner-sphere reorganization energy $\lambda_{i}$ (see values in Table S2).

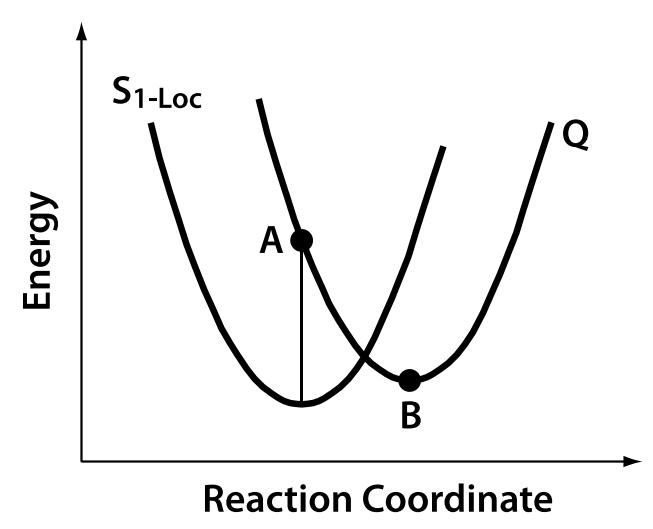

Scheme S3. Diagram showing geometry points used to estimate inner-sphere reorganization energy $\lambda_{i}$ for SF reaction in BT1. See S.I. and manuscript text for details. 
Table S2. Lowest energy quintet (Q) state energies (gas-phase calculations; 6-31g(d) and $\omega$ B97XD range corrected density functional) in fixed geometries relevant to the estimation of inner sphere reorganization $\lambda_{i}$ energy during SF. (see S.I. text for details about geometries used).

\begin{tabular}{cccc}
\hline Geometry & Energy/ Hartree & $\lambda_{\mathrm{i}} /$ Hartree & $\lambda_{\mathrm{i}} / \mathrm{eV}$ \\
\hline $\mathrm{S}_{1-\text { Loc }}($ geometry A) & -1500.02590004 & \multirow{2}{*}{0.015826} & 0.431 \\
$\mathrm{Q}$ (geometry B) & -1500.04172621 & & \\
\hline
\end{tabular}


Table S3. Cartesian coordinates for Geometry A (optimized geometry of the $\mathrm{S}_{1-\mathrm{Loc}}$ state) that was determined in reference ${ }^{3}$ using TD-DFT with gradients, the 6-31g(d) basis, the $\omega$-B97XD range corrected density functional, and a polarizable continuum model parameterized for toluene.

\footnotetext{
C $-1.19864600002 .4627510000 \quad 0.7050730000$

C $0.0150310000 \quad 3.27050800001 .1405210000$

C $0.01503000003 .2705080000-1.1405210000$

C $0.0362420000 \quad 4.3261200000 \quad 0.0000000000$

$\mathrm{H} \quad 0.9456420000 \quad 4.9351820000 \quad 0.0000000000$

$\mathrm{H}-0.8489760000 \quad 4.9689340000 \quad 0.0000000000$

C $1.2188140000 \quad 2.4355370000 \quad 0.7223160000$

C $1.21881300002 .4355360000-0.7223170000$

$\mathrm{H} \quad 0.0145070000 \quad 3.6303630000 \quad 2.1697000000$

$\mathrm{H} \quad 0.0145060000 \quad 3.6303620000-2.1697010000$

C $-2.1594540000 \quad 1.7760300000-1.4169530000$

C -2.15945300001 .77603000001 .4169530000$

C $-3.17373700001 .0816150000-0.7188350000$

$\mathrm{H}-2.15875700001 .7685280000-2.5042210000$

C $-3.1737370000 \quad 1.0816160000 \quad 0.7188340000$

$\mathrm{H}-2.1587570000 \quad 1.7685290000 \quad 2.5042210000$

C $-4.2039270000 \quad 0.3856410000-1.3967680000$

C $-4.2039270000 \quad 0.3856400000 \quad 1.3967680000$

C $-5.2258050000-0.2988280000-0.7256540000$

$\mathrm{H}-4.2018190000 \quad 0.3862570000-2.4848690000$

C $-5.2258050000-0.2988270000 \quad 0.7256550000$

$\mathrm{H}-4.2018190000 \quad 0.3862580000 \quad 2.4848690000$

C $-6.2460460000-0.9795260000-1.3992620000$

C $-6.2460460000-0.97952600001 .3992620000$

C $-7.2827400000-1.6675930000-0.7173620000$

$\mathrm{H}-6.2476430000-0.9813840000-2.4873380000$

C $-7.2827400000-1.6675920000 \quad 0.7173630000$

$\mathrm{H}-6.2476430000-0.9813820000 \quad 2.4873380000$

$\mathrm{H}-8.3094580000-2.3472840000 \quad 2.4833750000$

C $-8.3097410000-2.3472500000 \quad 1.3962760000$

C $-9.3140190000-3.0108650000 \quad 0.6989110000$

C $-9.3140190000-3.0108650000-0.6989100000$

$\mathrm{H}-10.0987250000-3.5289020000 \quad 1.2415790000$

C $-8.3097410000-2.3472500000-1.3962760000$

$\mathrm{H}-10.0987250000-3.5289020000-1.2415790000$

$\mathrm{H}-8.3094580000-2.3472850000-2.4833750000$

$\begin{array}{llll}\text { C }-1.1986460000 & 2.4627510000 & -0.7050740000\end{array}$

C $5.2233120000-0.3096680000-0.7201030000$

C $4.1981630000 \quad 0.3828420000-1.4001830000$

C $5.2233130000-0.3096670000 \quad 0.7201030000$

C $6.2446400000-0.9942610000-1.4014830000$

C $3.19908700001 .0604370000-0.7243760000$

H $4.1991480000 \quad 0.3833230000-2.4882000000$

C 4.19816400000 .38284200001 .4001820000

C $6.2446410000-0.99426100001 .4014830000$

C $7.2519830000-1.6664560000-0.7208650000$

H $6.2449110000-0.9944350000-2.4895090000$

C $3.19908700001 .0604390000 \quad 0.7243760000$

C $2.16119500001 .7751670000-1.4289170000$

$\mathrm{H} \quad 4.1991490000 \quad 0.3833260000 \quad 2.4881990000$

H $6.2449090000-0.9944400000 \quad 2.4895090000$

C $7.2519830000-1.6664560000 \quad 0.7208640000$

C $2.1611950000 \quad 1.7751670000 \quad 1.4289170000$

$\mathrm{H} 2.16713900001 .7756540000-2.5161830000$

H $2.1671400000 \quad 1.7756560000 \quad 2.5161830000$

H $10.0673740000-3.5357920000 \quad 1.2469190000$

C $9.2782550000-3.0126100000 \quad 0.7151350000$

C $8.3013490000-2.36407000001 .4061860000$

C $9.2782550000-3.0126110000-0.7151340000$

H $8.2999750000-2.3631520000 \quad 2.4932140000$

C $8.3013480000-2.3640710000-1.4061860000$

$\mathrm{H} 10.0673730000-3.5357920000-1.2469180000$

H $8.2999710000-2.3631570000-2.4932140000$
}

Table S4. Cartesian coordinates for Geometry B (optimized geometry of the Q state) that was determined in reference ${ }^{3}$ using TD-DFT with gradients, the 6-31g(d) basis, the $\omega$-B97XD range corrected density functional, and a polarizable continuum model parameterized for toluene. 


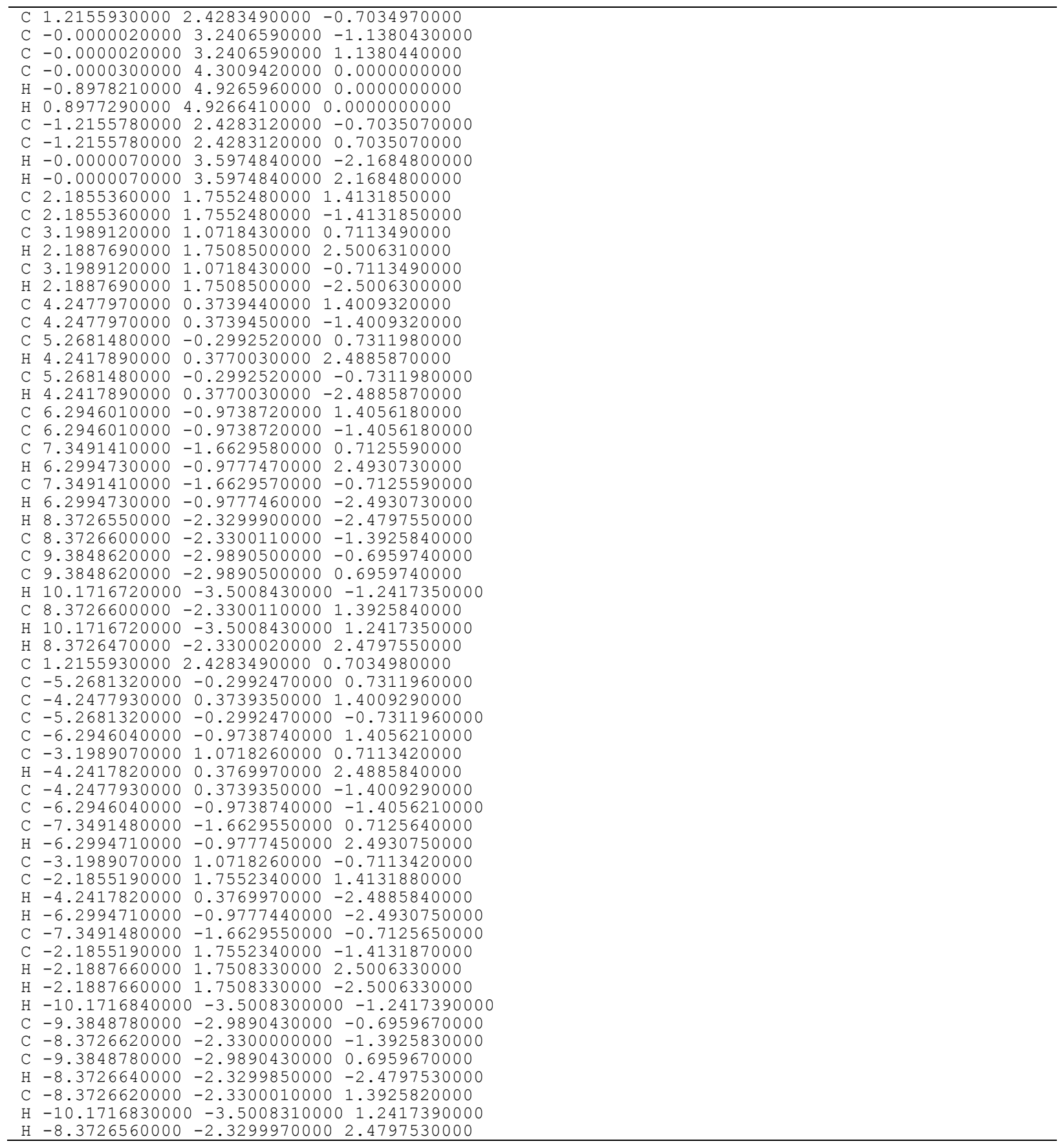

\section{References}

(1) Carey, T. J.; Snyder, J. L.; Michael, R. E.; Sammakia, T.; Damrauer, N. H. J.Org.Chem 2016, Submitted.

(2) Platt, J. R. J. Chem. Phys. 1949, 17, 484.

(3) Vallett, P. J.; Snyder, J. L.; Damrauer, N. H. J. Phys. Chem. A 2013, 117, 10824.

(4) Gaussian 09, R. D.; M. J. Frisch, G. W. T., H. B. Schlegel, G. E. Scuseria,; M. A. Robb, J. R. C., G. Scalmani, V. Barone, B. Mennucci,; G. A. Petersson, H. N., M. Caricato, X. Li, H. P. Hratchian,; A. F. Izmaylov, J. B., G. Zheng, J. L. Sonnenberg, M. Hada,; M. Ehara, K. T., R. Fukuda, J. Hasegawa, M. Ishida, T. Nakajima,; Y. Honda, O. K., H. Nakai, T. Vreven, J. A. Montgomery, Jr.,; J. E. Peralta, F. O., M. Bearpark, J. J. Heyd, E. Brothers,; K. N. Kudin, V. N. S., T. Keith, R. Kobayashi, J. Normand,; K. 
Raghavachari, A. R., J. C. Burant, S. S. Iyengar, J. Tomasi,; M. Cossi, N. R., J. M. Millam, M. Klene, J. E. Knox, J. B. Cross,; V. Bakken, C. A., J. Jaramillo, R. Gomperts, R. E. Stratmann,; O. Yazyev, A. J. A., R. Cammi, C. Pomelli, J. W. Ochterski,; R. L. Martin, K. M., V. G. Zakrzewski, G. A. Voth,; P. Salvador, J. J. D., S. Dapprich, A. D. Daniels,; O. Farkas, J. B. F., J. V. Ortiz, J. Cioslowski,; and D. J. Fox; Gaussian, Inc. : Wallingford, CT, 2009. 